\title{
REPRODUCIBILIDAD INTRAEXAMINADOR EN LA APLICACIÓN DEL SISTEMA INTERNACIONAL DE DETECCIÓN Y VALORACIÓN DE CARIES DENTAL (ICDAS II) *
}

\author{
${ }^{1}$ Mónica Bibiana Amaya Moreno, ${ }^{1}$ Camilo Andrés Villabona López, ${ }^{1}$ Oscar Javier Galvis Ramírez, ${ }^{1}$ Darlis Yaneth Santamaría Téllez, \\ ${ }^{2}$ Carmen Alodia Martínez López, ${ }^{3}$ Martha Juliana Rodríguez Gómez. \\ ${ }^{1}$ Estudiante X semestre F. de Odontología U Santo Tomás. ${ }^{2}$ Odontóloga U. Santo Tomás, Especialista en Ortopedia Maxilar U. Antonio Nariño, \\ Lic. Química y Biología U. Pedagógica y Tecnológica de Colombia, Docente U. Santo Tomás. ${ }^{3}$ Odontóloga U. Javeriana, \\ Especialista en Odontopediatría y Ortodoncia Preventiva U. CES, Docente U. Santo Tomás.
}

\section{RESUMEN}

Autor responsable de correspondencia: Carmen Alodia Martínez L.

Correo electrónico: aidola82@hotmail.com

Objetivo: Evaluar la reproducibilidad intraexaminador en la aplicación del Sistema Internacional de Valoración y Detección de Caries Dental (ICDAS II).

Materiales y métodos: Se realizó un estudio de evaluación diagnóstica: reproducibilidad - concordancia intraobservador. Un examinador evaluó 228 superficies dentales en 19 estudiantes de primer semestre de la Facultad de Odontología, de la Universidad Santo Tomás, a los que se les había realizado una profilaxis previa. Se evaluaron los siete criterios del ICDAS II (0 al 6). Los registros se realizaron en dos sesiones en el mismo horario, con un lapso de ocho días. Se calcularon las medidas de resumen según la naturaleza de la variable y se analizó la reproducibilidad (valor Kappa) a nivel global y de acuerdo con cada uno de los códigos establecidos.

Resultados: Se obtuvo un Kappa globlal de 0.7398. En relación con cada uno de los códigos se observó mayor consistencia en el diagnóstico de lesiones con decoloración café $(2 \mathrm{~B})$ con un $\mathrm{K}=0.8455$, y menor consistencia en el diagnóstico de la opacidad blanca al secar (1W) con un $\mathrm{K}=0.0066$.

Conclusión: La reproducibilidad global intraexaminador de los criterios ICDAS fue considerada como buena. Sin embargo, lo mismo no ocurrió al valorar las lesiones de mancha blanca (códigos 1 y 2); se requiere más entrenamiento para lograr una buena reproducibilidad en cada uno de los estadíos de la lesión. [Amaya MB, Villabona CA, Galvis OJ, Santamaria DY, Martínez CA, Rodríguez MJ. Reproducibilidad intraexaminador en la aplicación del Sistema Internacional de Detección y Valoración de Caries Dental (ICDAS II). Ustasalud 2009; 8: 19 - 24]

Palabras clave: Reproducibilidad, ICDAS II, Caries dental, Valor Kappa.

\section{INTRA-EXAMINER REPRODUCIBILITY IN THE INTERNATIONAL CARIES DETECTION AND ASSESSMENT SYSTEM (ICDAS II)}

\begin{abstract}
Objective: To assess intra-examiner reproducibility in the International Caries Detection and Assessment System (ICDAS II). Material and methods: A study of diagnostic reproducibility - agreement intra-examiner was done. An examiner evaluated 228 dental surfaces in 19 first semester students at the Dental School in the Santo Tomas University. The subjects' teeth were cleaned by brushing and then examined. Scoring for lesion was done using a seven-category scale (0-6) according to the ICDAS criteria. The exams were done in two sessions at the same schedule. Weighted kappa values for intra-examiner reproducibility were calculated, both global and for all the individual ICDAS-II-codes.

Results: The weighted Kappa values for the intra-examiner reproducibility were 0.7398 . There was a Kappa value of 0.8455 in the $2 \mathrm{~B}$ code (brown discoloration) and no agreement in the $1 \mathrm{~W}$ code (first white change in enamel visible after 5 seconds air-drying) with a Kappa value of 0.0066 .

Conclusion: The intra-examiner reproducibility was considered good for the ICDAS criteria. Besides, there was a poor and a moderate agreement in the $1 \mathrm{~W}$ and $2 \mathrm{~W}$ scores, white opacity after air-drying and white opacity without air-drying, respectively. Further training is required to achieve a good reproducibility in every score.
\end{abstract}

Key words: Reproducibility, ICDAS II, Dental caries, Kappa values.

Recibido para publicación: 20 de abril de 2009. Aceptado para publicación: 2 de julio de 2009 .

* Grupo de Investigación CYDUSTA. 


\section{INTRODUCCIÓN}

La caries dental describe los resultados de la disolución química localizada de la estructura dental debido a los procesos metabólicos que se desarrollan dentro de la placa bacteriana que cubre dicha superficie. ${ }^{1,2}$ Esto implica que la enfermedad sea considerada como un fenómeno natural que ocurre en cualquier lugar del tejido dentario en el que la placa bacteriana permanezca algún tiempo sin removerse. ${ }^{3}$ Por lo tanto, las zonas en las que es más difícil realizar una limpieza adecuada se consideran más propensas; éstas son las fosas y fisuras especialmente durante la erupción dental, las zonas cervicales interproximales por debajo del punto de contacto y a lo largo del margen gingival, y las superficies retentivas (obturaciones desadaptadas, aparatos ortodóncicos). ${ }^{4}$

Al considerar la caries dental como un proceso es necesario que su diagnóstico sea coherente con los eventos que están sucediendo en la estructura dental. Es así como el diagnóstico no sólo implica la detección de la lesión sino que también debe determinar su severidad y su actividad. ${ }^{4}$ En las últimas décadas, se han hecho esfuerzos en la consecución de estrategias diagnósticas que guíen a una adecuada toma de decisiones para la planeación de tratamientos más conservadores de acuerdo con el estadio en que se encuentra la lesión de caries dental.
Durante el examen clínico del paciente, el método visual táctil es considerado uno de los más usados, rápidos y fáciles para valorar las superficies coronales, aunque sea difícil de monitorear. ${ }^{5}$ Dentro de esta categoría se encuentra el enfoque de la Organización Mundial de la Salud que plantea el diagnóstico de lesiones de caries dental a partir de la cavitación, ${ }^{6}$ lo que niega la oportunidad de realizar intervenciones no operatorias. En 1988, Pitts y Fiffe presentaron una clasificación en la que tuvieron en cuenta tanto lesiones cavitacionales como no cavitacionales; ${ }^{7}$ sin embargo, no establecía su actividad.

Ekstrand y colaboradores (1997) relacionaron los diferentes estadios clínicos con la profundidad histológica de la lesión en molares y premolares extraídos. ${ }^{8}$ Por su parte, Nyvad y colaboradores, en 1999, describieron una serie de criterios diagnósticos que diferenciaban tanto las lesiones activas de las inactivas como las cavitacionales de las no cavitacionales. ${ }^{9}$

Debido al avance en la investigación, en 2002 tuvo lugar el International Consensus Workshop on Caries Clinical Trials (ICW-ICCT) cuyo objetivo fue crear un sistema estandarizado basado en la evidencia para mejorar la toma de decisiones acerca del diagnóstico, pronóstico y plan de tratamiento adecuado tanto a nivel individual como público. A partir de esta reunión se inicio el desarrollo del Sistema Internacional de Valoración y Detección de Caries Dental (ICDAS) (Tabla 1). ${ }^{5,10}$

Tabla 1. Sistema Internacional de Detección y Valoración de Caries (ICDAS II). ${ }^{11}$

\begin{tabular}{cl}
\hline Código & \multicolumn{1}{c}{ Descripción clínica } \\
\hline 0 & $\begin{array}{l}\text { Sano. } \\
\text { No hay evidencia de caries después de secar por } 5 \text { segundos. }\end{array}$ \\
& $\begin{array}{l}\text { Primer cambio visual en el esmalte. } \\
\text { No hay evidencia de cambio de color en húmedo, al secar se observa una coloración blanca que no es } \\
\text { consistente con el esmalte sano. } \\
\text { Decoloración café confinada a la fisura/angosta en la superficie lisa (se observa en húmedo o seco) }\end{array}$ \\
& $\begin{array}{l}\text { Cambio visual evidente en el esmalte. } \\
\text { Se observa una decoloración blanca en húmedo. } \\
\text { Decoloración café más allá de la fisura/amplia la en superficie lisa. }\end{array}$ \\
3 & $\begin{array}{l}\text { Ruptura localizada del esmalte sin dentina visible o sombra subyacente. } \\
4\end{array}$ \\
5 & Sombra subyacente con o sin ruptura localizada del esmalte. \\
& Cavidad detectable con dentina visible. \\
& coronal.
\end{tabular}


Los criterios del ICDAS se han podido usar como una herramienta de fácil manejo y alta confiabilidad para el diagnóstico temprano de la caries dental. Actualmente, están siendo aplicados en varias facultades de odontología a nivel mundial, en centros de investigación y a nivel gubernamental. ${ }^{12-14}$ Adicionalmente, se han realizado varios estudios para evaluar su reproducibilidad inter e intraexaminador. ${ }^{15-18}$

Jablonsski-Momeni y colaboradores (2008) demostraron reproducibilidad y precisión diagnóstica al usar ICDAS II en 100 molares extraídos, en las diferentes etapas del proceso carioso. Los valores Kappa estuvieron entre 0.62 y 0.83 para los examinadores evaluados. ${ }^{15}$

En Colombia, se han realizado varios estudios de calibración por un examinador entrenado (Examinador Estándar). Se realizan conferencias sobre los conceptos actuales de caries dental, explicaciones sobre los códigos ICDAS, discusiones de casos clínicos, exámenes preclínicos en dientes naturales y exámenes clínicos en pacientes. ${ }^{12} \mathrm{Al}$ evaluar 69 examinadores, $19 \%$ obtuvieron un nivel aceptable de acuerdo (Kappa 0.60). ${ }^{16}$

El propósito de este estudio fue evaluar la reproducibilidad intraexaminador en la aplicación de los criterios del ICDAS II en el diagnóstico visual de caries dental en las clínicas odontológicas de la Universidad Santo Tomás, en Bucaramanga.

\section{MATERIALES Y METODOS}

Se realizó un estudio de evaluación de tecnología diagnóstica: reproducibilidad - concordancia in- traobservador, para evaluar el nivel de acuerdo en los códigos del ICDAS II por un examinador docente, vinculado con la Facultad de Odontología de la Universidad Santo Tomás previamente capacitado por un experto en el manejo de los criterios mencionados.

El universo lo conformaron 131 estudiantes de I semestre de la Facultad de Odontología de la Universidad Santo Tomás. La muestra estuvo constituida por 19 estudiantes. En cada estudiante se evaluaron doce superficies dentales de forma no aleatoria por conveniencia, entre vestibular, oclusal y palatina, para un total de 228 superficies.

En el estudio se incluyeron los estudiantes matriculados en I semestre en la Facultad de Odontología, durante primer periodo del 2008, que aceptaron participar en el estudio y cuyas superficies dentales estuvieran libres de restauraciones y sellantes. Dentro de los criterios de exclusión estaban: mujeres en estado de gestación, estudiantes que se encontraran en tratamiento de ortodoncia y aquellos que no desearan participar en la investigación.

Se registraron las variables sociodemográficas como género y edad, y variables para evaluar el nivel global de reproducibilidad intraevaluador: ICDAS II-1 (primera sesión), ICDAS II-2 (segunda sesión). Para las variables orientadas a evaluar el nivel de reproducibilidad según el código ICDAS II se adicionaron las letras W (opacidad blanca) y B (decoloración café) (Tabla 2).

Tabla 2. Sistema Internacional de Detección y Valoración de Caries usado en el procedimiento.

\begin{tabular}{cl}
\hline Código & \\
\hline 0 & $\begin{array}{l}\text { Sano. } \\
\text { No hay evidencia de caries después de secar por } 5 \text { segundos. } \\
\text { Primer cambio visual en el esmalte. }\end{array}$ \\
1W & $\begin{array}{l}\text { No hay evidencia de cambio de color en húmedo, al secar se observa una opacidad blanca que no es } \\
\text { consistente con el esmalte sano. }\end{array}$ \\
1B & $\begin{array}{l}\text { Primer cambio visual en el esmalte. } \\
\text { Decoloración café confinada a la fisura/angosta en la superficie lisa. }\end{array}$ \\
2W & $\begin{array}{l}\text { Cambio visual evidente en el esmalte. } \\
\text { Se observa una opacidad blanca en húmedo. }\end{array}$ \\
2B & $\begin{array}{l}\text { Cambio visual evidente en el esmalte. } \\
\text { Decoloración café más allá de la fisura/amplia la en superficie lisa. }\end{array}$ \\
4 & Ruptura localizada del esmalte sin dentina visible o sombra subyacente. \\
5 & Sombra subyacente con o sin ruptura localizada del esmalte. \\
6 & Cavidad detectable con dentina visible.
\end{tabular}




\section{Procedimiento.}

La medición de la reproducibilidad se hizo a partir de dos sesiones de examen con un intervalo de ocho días. El estudio se llevó a cabo en las clínicas odontológicas de la Universidad Santo Tomás. Se realizó una prueba piloto con la participación de cuatro estudiantes de décimo semestre con el fin de evaluar el instrumento y probar el diseño metodológico.

Cada estudiante participante fue informado de manera verbal y escrita sobre el objetivo del estudio. Aquellos que desearon ser incluidos firmaron el consentimiento informado para proceder al examen inicial. Antes de éste, se realizó una profilaxis con copa caucho y se establecieron las superficies dentales que serían valoradas por el examinador docente.

Se establecieron horarios únicos comprendidos entre las 7:00 a.m. a 9:00 a.m., y de 10:00 a.m. a 12:00 $\mathrm{m}$. En cada una de las sesiones se usó el campo fenestrado para asegurar la privacidad de la persona evaluada y cada estudiante fue sometido a limpieza mecánica con cepillo profiláctico montado en una pieza de mano de baja velocidad, piedra pómez y crema dental. El examinador docente utilizó la misma unidad odontológica durante las dos sesiones del estudio, bajo la misma luz a la distancia que consideró adecuada. El examen fue realizado con un instrumental básico para el diagnóstico de la caries dental que estuvo conformado por una sonda periodontal 11.5B (Hu-Friedy ${ }^{\circledR}$ ) y espejo bucal \# 5 . Adicionalmente, se usó la jeringa triple y rollos de algodón si se requería. El tiempo para evaluar cada superficie no superó los 30 segundos.

Los datos registrados fueron consignados por duplicado tal y como se dictaron en una libro Excel diseñado para tal fin. Éstos se exportaron a la base de datos Epi - Info. 6.04 y se verificó la digitación, luego se llevaron al STATA 8.0 para su correspondiente análisis. ${ }^{19-21}$

\section{Plan de análisis estadístico}

\section{Análisis univariado}

Se calcularon medidas de resumen según la naturaleza de la variable:

Variables cualitativas:

- Proporción y razón:

Género paciente

Variables cuantitativas:

- Tendencia central: media, mediana, moda. Dispersión: rango, desviación estándar, varianza.

- Edad del estudiante.

\section{Análisis de reproducibilidad}

Se calcularon las pruebas estadísticas de acuerdo con la asociación de variables:

\section{Test de Kappa ponderado global}

- Reproducibilidad según ICDAS1 / Reproducibilidad ICDAS2

\section{Test de Kappa para cada una de las superfi-} cies según el criterio

- 0 (1) / 0 (2)

- $1 \mathrm{~W}(1) / 1 \mathrm{~W}(2)$

- $1 \mathrm{~B}(1) / 1 \mathrm{~B}(2)$

- $2 \mathrm{~W}(1) / 2 \mathrm{~W}(2)$

- $2 \mathrm{~B}(1) / 2 \mathrm{~B}(2)$

- 3 (1) / 3 (2)

- 4 (1) / 4 (2)

- $5(1) / 5(2)$

- 6 (1) / 6 (2)

Se dispuso de la escala presentada por Altman para la interpretación del valor $\mathrm{K}$ (Tabla 3). ${ }^{22}$

Tabla 3. Interpretación del valor Kappa (K). ${ }^{22}$

\begin{tabular}{cc}
\hline Valor $\mathbf{K}$ & Fuerza de la Concordancia \\
\hline$<0.20$ & Pobre \\
$0.21-0.40$ & Débil \\
$0.41-0.60$ & Moderada \\
$0.61-0.80$ & Buena \\
$0.81-1.00$ & Muy buena \\
\hline
\end{tabular}

\section{Consideraciones éticas}

Al tener en cuenta las recomendaciones establecidas en la Resolución 008430 de 1993 del Ministerio de Protección Social de la República de Colombia, se consideró el Artículo 8 del Título II al proteger la privacidad de los individuos que se encuentren participando en el estudio. Este estudio no representó ningún tipo de riesgo debido a que fue un estudio de técnica diagnóstica donde no se modificó ninguna variable biológica, fisiológica y psicológica de los pacientes ni de la muestra (Artículo 11, Título II). ${ }^{23}$

\section{RESULTADOS}

\section{Análisis univariado.}

\section{Variables sociodemográficas}

Se evaluaron 19 (100\%) estudiantes de I semestre de la Facultad de Odontología. El 63.2\% (12) eran mujeres, con una edad promedio global de $17+$ 


\section{4 años (Tabla 4).}

Tabla 4. Descripción de la población evaluada.

\begin{tabular}{cc}
\hline Género & $\begin{array}{c}\text { Global } \\
\mathbf{n}(\%)\end{array}$ \\
\hline Mujeres & $12(63.2)$ \\
Hombres & $7(36.8)$ \\
Edad (promedio) & $17.4+1.4$ \\
\hline
\end{tabular}

\section{Nivel global de reproducibilidad intraevaluador.}

Se realizó un análisis global de concordancia Kappa ponderado, donde se registró un $\mathrm{K}=0.7398$, considerado como bueno (Tabla 5).

Tabla 5. Análisis del nivel global de reproducibilidad intraevaluador según los criterios del ICDAS.

\begin{tabular}{ccc}
\hline Kappa & Std. Err & Prob \\
\hline 0.7398 & 0.0461 & $<0.001$ \\
\hline
\end{tabular}

\section{Nivel de reproducibilidad según los criterios del ICDAS}

Al analizar la reproducibilidad de cada uno de los criterios del ICDAS, se observó mayor consistencia en el código $2 \mathrm{~B}$ con un $\mathrm{K}=0.8455$ y en el código $1 \mathrm{~B}$ con un $\mathrm{K}=0.8313$; su concordancia fue Muy Buena.

Se observó menor consistencia intraexaminardor en el código $1 \mathrm{~W}$ con un $\mathrm{K}=0.0066$. Su concordancia fue pobre. En la muestra, no se presentaron códigos 4 y 6 (Tabla 6).

Tabla 6. Análisis del nivel de reproducibilidad según cada uno de los códigos del ICDAS.

\begin{tabular}{ccc}
\hline Código & Kappa & Error Estándar \\
\hline 0 & 0.7694 & 0.0659 \\
$1 \mathrm{~W}$ & 0.0066 & 0.0572 \\
$1 \mathrm{~B}$ & 0.8313 & 0.0662 \\
$2 \mathrm{~W}$ & 0.5679 & 0.0649 \\
$2 \mathrm{~B}$ & 0.8455 & 0.0654 \\
3 & 0.6156 & 0.0661 \\
4 & - & - \\
5 & 0.7979 & 0.0649 \\
6 & - & - \\
\hline
\end{tabular}

\section{DISCUSIÓN}

El objetivo de este trabajo fue evaluar la reproducibilidad intraexaminador de los criterios del ICDAS en 228 superficies dentales de 19 estudiantes de I semestre de la Facultad de Odontología de la Universidad Santo Tomás en Bucaramanga. El resultado globlal se considero como Bueno (Kappa ponderado $=0.7398$ ) de acuerdo con la Interpretación del Valor Kappa. ${ }^{22}$

Es de anotar que el odontólogo se considera calibrado para realizar diagnóstico con los criterios ICDAS cuando la concordancia interexaminador e intraexaminador alcancen un nivel aceptable de $\mathrm{K}>0.60 .^{12}$ La reproducibilidad intraexaminador evaluada en este estudio alcanzó este nivel.

El valor obtenido en el presente estudio fue similar al valor Kappa obtenido por el examinador en el IV Curso Teórico - Práctico: Concordancia en el Diagnóstico Visual de Caries Dental Criterios ICDAS (2007) que fue de 0.685.

Este resultado no difiere del obtenido por Shoaib y colaboradores que examinaron 112 dientes. En ese estudio, la reproducibilidad intraexaminador fue de 0.72 a $0.85 .{ }^{17}$ Así mismo, Jablonski-Momeni y colaboradores encontraron una reproducibilidad intraexaminador de 0.73 a $0.83 .{ }^{15}$ Estos dos estudios fueron realizados in vitro.

Por otra parte, Braga y colaboradores realizaron un estudio en 252 niños de 36 a 59 meses de edad. Obtuvieron una reproducibilidad intraexaminador de 0.60 a 0.79 en el entrenamiento realizado en dientes extraídos. Sin embargo, no se evaluó la reproducibilidad intraexaminador en la valoración realizada a los niños que conformaron la muestra ya que requería que el menor fuera examinado por segunda vez y no representaba el objetivo del estudio. $^{18}$

Adicionalmente, en este estudio se presentaron diferencias en los valores Kappa al considerar cada uno de los criterios. Al analizar los códigos de las lesiones no cavitacionales (1W, 1B, 2W, 2B) se obtuvo una Muy Buena concordancia en los criterios $1 \mathrm{~B}$ y $2 \mathrm{~B}$, con unos valores $\mathrm{K}$ de 0.8313 y de 0.8455 , respectivamente. En contraste, hubo una Pobre concordancia en el criterio $1 \mathrm{~W}(\mathrm{~K}=0.0066)$. Es claro que este hallazgo se debió a que las lesiones de mancha café son más evidentes que las lesiones de mancha blanca.

Estos resultados coinciden con el reporte de la experiencia de tres ejercicios de la calibración conducidos en Colombia por un examinador entrenado (Examinador Estándar) que concluyó que el 19\% de los examinadores alcanzaron niveles aceptables con un $\mathrm{K}=0.60$ y que la mayor parte de los errores se presentaron al diagnosticar el criterio $1 \mathrm{~W}$ con sólo el $28.7 \%$ de acuerdo. ${ }^{16}$ 
Una de las fortalezas del estudio fue continuar con la difusión de los criterios ICDAS y mostrar que se requiere un mayor estudio y entrenamiento para realizar un adecuado diagnóstico de caries dental que implique no sólo la valoración de la superficie sino también la planeación correcta del tratamiento ya sea preventivo u operatorio. Dentro de las debilidades, se puede citar la dificultad para encontrar reportes completos en la literatura que evalúen la reproducibilidad de los criterios.

El Sistema Internacional de Valoración y Detección de Caries (ICDAS) se considera una herramienta de gran utilidad para sensibilizar a los profesionales de la salud sobre la importancia de manejar la caries dental como un proceso que puede ser controlado de tal manera que se traten las lesiones de una manera menos operatoria y más preventiva. ${ }^{24}$ Es por eso que se requiere un mayor entrenamiento para aplicarlos adecuadamente.

\section{BIBLIOGRAFÍA}

1. Fejerskov O, Kidd EAM, Nyvad B, Baelum V. Defining the Disease: An Introduction. En: Fejerskov O, Kidd EAM. Dental Caries: Disease and its Clinical Management. Singapore: Blackwell Munksgaard; 2008. p. 4-6.

2. Ekstrand KR. Improving clinical visual detection - potencial for caries clinical trials. J Dent Res 2004; 83 (Special Iss C): C67-C71.

3. Manji, F, Fejerskov O, Nagelkerke NJD, Baelum V. A ramdom effects model for some epidemiological features of dental caries. Community Dent Oral Epidemiol 1991; 19:324-328.

4. Kidd EAM, Fejerskov O. What constitutes dental caries? Histopathology of carious enamel and dentin related to the action of cariogenic biofilms. J Dent Res 2004; 83 (Special Iss C): C35-C38.

5. Pitts NB. "ICDAS" - An international system for caries detection and assessment being developed to facilitate caries epidemiology, research and appropriate clinical management. Community Dental Health 2004; 21: 193-198.

6. World Health Organization. Oral Health Surveys: Basic Methods. 4th. Edition. Geneva: WHO, 1997.

7. Pitts NB, Fyffe HE. The effect of varying diagnostic thresholds upon clinical caries data for a low prevalence group. J Dent Res 1988; 67: 591-596.

8. Ekstrand KR, Ricketts DN, Kidd EA. Reproducibility and accuracy of the methods for assessment of demineralization depth on the occlusal surface: An in vitro examination. Caries Res 1997; 31: 224-231.

9. Nyvad B, Machiulskiene V, Baelum V. Reliability of a new caries diagnostic system differentiating between active and inactive caries lesions. Caries Res 1999; 33: 252-260.

10. Martignon S, Zarta OL. Nuevos horizontes en prevención y operatoria: Alternativas del proceso diagnostico. Revista Científica de la Facultad de Odontología 2000; 6: 2.
11. International Caries Detection and Assessment System (ICDAS) Coordinating Committee. Criteria Manual - International Caries Detection and Assessment System (ICDAS II). Scotland: Dental Health Services Research Unit; 2005. URL disponible en: http://www.icdas.org

12. Martignon S, Tellez M. Criterios ICDAS: Nuevas perspectivas para el diagnóstico de la caries dental. Revista Dental Main News 2007; 14-19.

13. Ismail AI, Sohn W, Tellez M, Amaya A, Sen A, Hasson $\mathrm{H}$, Pitts NB. The International Caries Detection and Assessment System (ICDAS): an integrated system for measuring dental caries. Community Dent Oral Epidemiol 2007; 35: 170-178.

14. Martignon S, González M, McCornikc V, Ruiz JA, Jácome S, Guarnizo C. Guía de Diagnóstico, Prevención y Tratamiento de la Caries Dental. Asociación Colombiana de Facultades de Odontología - ACFO., Secretaria Distrital de Salud Bogotá. Editor: Secretaria Distrital de Salud Bogotá, Bogotá, 2007.

15. Jablonsski-Momeni A, Stachniss V, Ricketts DN, HeinzelGutenbrunner M, Pieper K. Reproducibility and accuracy of the ICDAS-II for detection of occlusal caries in vitro. Caries Res 2008; 42: 79-87.

16. Gamboa LF, Gil F, Martignon S. Experience of calibration exercises conducted in Colombia with the ICDAS II visual criteria for primary coronal caries. Caries Res 2007; 41 [Abstract]

17. Shoaib L, Deery C, Nugent ZJ, Ricketts DNJ. Reproducibility of ICDAS II criteria for occlusal y and approximal caries detection in primary teeth. Caries Res 2007; 41 [Abstract]

18. Braga MM, Oliveira LB, Bonini GAVC, Bönecker M, Mendes FM. Feasibility of the International Caries Detection and Assessment System (ICDAS II) in the epidemiological survey and comparability with standard World Health Organization criteria. Caries Res 2009; 43: 245-249.

19. Microsoft Office Excel. Microsoft Corporation, 2007.

20. CDC - OMS. Epi - info 6 version 6.04a noviembre 1996.

21. Stata Corp 2003. Stata Statistical Software: Release 8.0. collage sation, TX; stata Corporation.

22. Altman DG. Practical statistics for medical research. New York: Chapman and Hall; 1991.

23. República de Colombia. Ministerio de Salud. Resolución No. 008430 (Octubre 4 1993); Bogotá.

24. Pitts NB. Are we ready to move from operative to non operative/preventive treatment of dental caries in clinical practice. Caries Res 2004; 38: 294-304. 